\title{
Preclinical evaluation of degradation kinetics and elemental mapping of first- and second-generation bioresorbable magnesium scaffolds
}

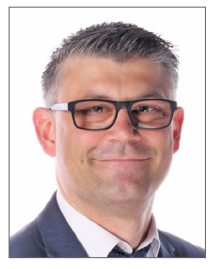

Michael Joner ${ }^{1 *}$, MD; Philipp Ruppelt ${ }^{2}$, PhD; Philine Zumstein ${ }^{2}$, MSc; Capucine Lapointe-Corriveau³, MSc; Guy Leclerc ${ }^{3,4}$, MD; Anna Bulin, DVM; Maria Isabel Castellanos ${ }^{1}, \mathrm{PhD}$; Eric Wittchow ${ }^{5}$, PhD; Michael Haude ${ }^{6}, \mathrm{MD}$; Ron Waksman ${ }^{7}, \mathrm{MD}$

1. Deutsches Herzzentrum München und Deutsches Zentrum für Herz-Kreislaufforschung e.V., Munich, Germany;

2. BIOTRONIK AG, Bülach, Switzerland; 3. AccelLAB Inc., Boisbriand, Quebec, Canada; 4. University of Montreal, Montreal, Quebec, Canada; 5. CVPath Institute, Inc., Gaithersburg, MD, USA; 6. Städtische Kliniken Neuss, Lukaskrankenhaus GmbH, Neuss, Germany; 7. MedStar Washington Hospital Center, Washington, DC, USA

This paper also includes supplementary data published online at: http://www:pcronline.com/eurointervention/142nd_issue/187

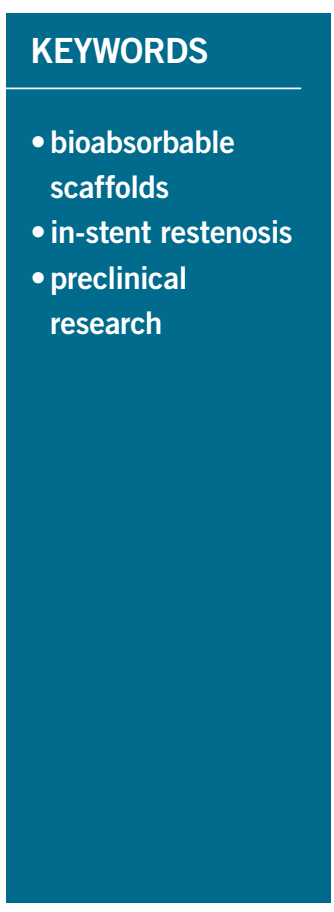

\begin{abstract}
Aims: Because vascular restoration therapy using bioresorbable vascular scaffolds (BRS) remains an appealing concept to restore vasoreactivity, an understanding of biodegradation remains paramount during preclinical testing. We therefore aimed to investigate the qualitative and temporal course of degradation of magnesium alloy-based bioresorbable vascular scaffolds in juvenile swine.

Methods and results: Qualitative characterisation of biodegradation was performed in 41 DREAMS $1 \mathrm{G}$ up to three years, while degradation kinetics were acquired in 54 DREAMS $2 \mathrm{G}$ implanted into porcine coronary arteries for 28, 90 and 180 days, one and two years. Assessment of end product composition was achieved in DREAMS $2 \mathrm{G}$ at 180 days. Myocardium was examined, while an OCT attenuation score was derived at strut level from 180 days to two years in DREAMS 2G. Degradation of DREAMS entails two corrosive phases. At one year, $94.8 \%$ of the magnesium was bioabsorbed in DREAMS $2 \mathrm{G}$ and, at two years, magnesium was completely replaced by amorphous calcium phosphate. Von Kossa staining revealed variable peri-strut mineralisation at all time points and only small focal myocardial emboli observed in one animal in the 180 days cohort. Strut discontinuity density was low at 28 days $(0.5 \pm 0.57$ per $\mathrm{mm})$ and increased to a density above 7.5 per $\mathrm{mm}$ up to one year. OCT attenuation score correlated well with strutbased degradation analysis up to two years.
\end{abstract}

Conclusions: While the current set of data supports vascular safety, clinical trials are warranted to prove the concept of vascular restoration following DREAMS implantation.

\footnotetext{
*Corresponding author: German Heart Center Munich, Lazarettstrasse 36, 80636 Munich, Germany. E-mail:joner@dhm.mhn.de
} 


\section{Abbreviations}

$\begin{array}{ll}\text { ACP } & \text { amorphous calcium phosphate } \\ \text { BRS } & \text { bioresorbable vascular scaffold } \\ \text { DES } & \text { drug-eluting stent } \\ \text { DREAMS } & \text { drug-eluting bioresorbable metal scaffold } \\ \text { EDX } & \text { energy dispersive X-ray analysis } \\ \text { GLMM } & \text { generalised linear mixed regression model } \\ \text { ICC } & \text { intra-class correlation coefficient } \\ \text { OCT } & \text { optical coherence tomography } \\ \text { PCI } & \text { percutaneous coronary intervention } \\ \text { PMMA } & \text { polymethyl methacrylate } \\ \text { SEM } & \text { scanning electron microscopy } \\ \text { XRD } & \text { X-ray diffraction } \\ \text { HCT } & \text { micro-computed tomography }\end{array}$

\section{Introduction}

Despite the compelling evidence of DES efficacy in a large proportion of patient and lesion subsets ${ }^{1}$, important limitations remain such as hypersensitivity reactions, late stent thrombosis and progression of atherosclerosis within the stented arterial segment (i.e., neoatherosclerosis $)^{2,3}$. Vascular restoration therapy was introduced as a disruptive concept to overcome some of these limitations by enabling transient mechanical vessel support following balloon dilatation with the help of bioresorbable scaffold implantation.

BIOTRONIK's magnesium scaffold (DREAMS 2G, Magmaris ${ }^{\circledR}$; BIOTRONIK, Bülach, Switzerland), a drug-eluting metallic bioresorbable scaffold, was developed as an improved iteration of its predecessor model DREAMS $1 \mathrm{G}$ to meet the above-mentioned goals and is the first CE-marked metallic bioresorbable scaffold. While previous preclinical studies addressed the biodegradation kinetics and vascular reactions during breakdown of polymeric $\mathrm{BRS}^{4}$, little is known with regard to qualitative and quantitative degradation of magnesium scaffolds. Furthermore, the fundamental disparity of biodegradation pathways among polymeric and magnesium scaffolds makes dedicated studies addressing these differences indispensable. In a prior study by Wittchow et al, general aspects of magnesium degradation were reported; however, few details were provided with regard to the dynamics of biodegradation and the quality of end products ${ }^{5}$. The change of drug-carrying polymer matrix from DREAMS $1 G$ (PLGA and paclitaxel) to $2 \mathrm{G}$ (PLLA and sirolimus) further warrants characterisation of biodegradation in DREAMS $2 \mathrm{G}$ with respect to its qualitative aspects and as a function of time.

\section{Methods}

\section{STUDY FLOW AND TEST DEVICES}

DREAMS $1 \mathrm{G}$ refers to a drug-eluting bioresorbable metal scaffold (BIOTRONIK) consisting of a balloon-expandable delivery system carrying a $3.0 \times 16 \mathrm{~mm}$ proprietary magnesium alloy-based scaffold with a six-crown three-link design and square-shaped struts at a thickness and width of $120 / 140 \mu \mathrm{m}$ coated with biodegradable poly-lactic-co-glycolic acid polymer and paclitaxel at a concentration of $7.4 \mu \mathrm{g} / \mathrm{cm}^{2}$.
DREAMS $2 \mathrm{G}$ refers to the most recent drug-eluting bioresorbable metal scaffold, consisting of a balloon-expandable delivery system carrying a $3.0 \times 20 \mathrm{~mm}$ proprietary magnesium alloy-based scaffold with a six-crown two-link design and square-shaped struts at a thickness and width of $150 / 150 \mu \mathrm{m}$ coated with degradable poly-L-lactic acid polymer and sirolimus at a concentration of $140 \mu \mathrm{g} / \mathrm{cm}^{2}$.

Uncoated DREAMS $2 \mathrm{G}$ refers to a bioresorbable metal scaffold (BIOTRONIK), consisting of a balloon-expandable delivery system carrying a $3.0 \times 15 \mathrm{~mm}$ or $3.0 \times 20 \mathrm{~mm}$ proprietary magnesium alloy-based scaffold with a six-crown two-link design and squareshaped struts at a thickness and width of $150 / 150 \mu \mathrm{m}$ without drug coating.

The current study includes results from two different generations of drug-eluting bioresorbable metal scaffold (DREAMS) owing to iterative device progress over time. Since qualitative aspects of degradation are identical for the described generations of BIOTRONIK's DREAMS, qualitative characterisation of magnesium backbone degradation was investigated in first-generation DREAMS (DREAMS 1G). For that purpose, 41 DREAMS $1 \mathrm{G}$ were implanted into the coronary arteries of 34 healthy Yucatan mini-swine. The following time points and animal/device numbers were applied: acute (4 animals/5 DREAMS 1G), 28 days $(8 / 8), 90$ days $(8 / 8), 180$ days $(8 / 8), 1$ year $(4 / 6), 2$ years $(1 / 3)$ and 3 years $(1 / 3)$.

To establish the time course of degradation (quantitative degradation) in contemporary second-generation Magmaris (DREAMS $2 \mathrm{G})$ drug-eluting bioresorbable metal scaffolds, a total of 54 DREAMS 2G were implanted into the coronary arteries of 50 Yucatan mini-swine. The following time points and animal/device numbers were applied: 28 days (12 animals/12 DREAMS 2G), 90 days (12/12), 180 days (12/12), 1 year (6/8), 2 years (8/10).

To investigate the crystal structure of degradation products, a total of three uncoated DREAMS $2 \mathrm{G}$ were implanted into the coronary arteries of two Goettingen mini-swine while three non-implanted and uncoated DREAMS $2 \mathrm{G}$ served as controls (Figure 1). The implanted devices were harvested after 180 days to ensure complete degradation of the scaffold.

Animal husbandry, medication administration, and scaffold implantation were performed according to standards as previously reported ${ }^{4}$.

Segments of all three main coronary arteries with favourable anatomy and diameter were selected by angiography at the discretion of the interventionalist and the devices were implanted targeting a balloon-to-artery ratio of 1.05 to 1.2 .

Study protocols were approved by the Institutional Animal Care and Use Committee of the testing facility (AccelLAB Inc., Boisbriand, Quebec, Canada) and the state administration of Saxony-Anhalt (Germany, 42502-3-763 IMTR) and followed the requirements and guidelines of the commission directive 86/609/ EEC, the German Animal Protection Act (version May 18, 2006, amended on July 28, 2014) and the Canadian Council on Animal Care regulations. 


\begin{tabular}{|c|c|c|c|}
\hline & $\begin{array}{c}\text { Time course of degradation } \\
\text { and calcification: } \\
50 \text { pigs implanted with DREAMS } 2 \mathrm{G}\end{array}$ & $\begin{array}{l}\text { Qualitative degradation process: } \\
34 \text { pigs implanted with DREAMS } 1 G\end{array}$ & $\begin{array}{c}\text { Crystallinity of degradation product: } \\
2 \text { pigs implanted } \\
\text { with uncoated DREAMS } 2 \mathrm{G}\end{array}$ \\
\hline Acute & & $\begin{array}{l}4 \text { Yucatan mini-swine ( } 5 \text { devices): } \\
\text { high res. } \mu C T(3)+E D X(5)\end{array}$ & \\
\hline 28 days & $\begin{array}{l}12 \text { Yucatan mini-swine (12 devices): } \\
\text { planimetry (12) + low res. } \mu C T(12)+ \\
\text { von Kossa (12) }\end{array}$ & $\begin{array}{l}8 \text { Yucatan mini-swine (8 devices): } \\
\text { high res. } \mu C T(3)+E D X(8)\end{array}$ & \\
\hline 90 days & $\begin{array}{l}12 \text { Yucatan mini-swine (12 devices): } \\
\text { planimetry (12) + low res. } \mu C T(12)+ \\
\text { von Kossa (12) }\end{array}$ & $\begin{array}{l}8 \text { Yucatan mini-swine (8 devices): } \\
\text { high res. } \mu C T(3)+E D X(8)\end{array}$ & \\
\hline 180 days & $\begin{array}{l}12 \text { Yucatan mini-swine (12 devices): } \\
\text { planimetry (12) + von Kossa (12) + } \\
\text { OCT (6) }\end{array}$ & $\begin{array}{l}8 \text { Yucatan mini-swine (8 devices): } \\
\text { high res. } \mu C T(3)+E D X(8)\end{array}$ & $\begin{array}{l}2 \text { Goettingen mini-swine (3 devices): } \\
X R D(3)\end{array}$ \\
\hline 1 year & $\begin{array}{l}6 \text { Yucatan mini-swine (8 devices): } \\
\text { planimetry }(8)+\text { low res. } \mu C T(8)+ \\
\text { von Kossa }(8)+\text { OCT }(4)\end{array}$ & $\begin{array}{l}4 \text { Yucatan mini-swine (6 devices): } \\
\text { high res. } \mu C T(3)+E D X(6)\end{array}$ & \\
\hline 2 years & $\begin{array}{l}8 \text { Yucatan mini-swine (10 devices): } \\
\text { planimetry }(10)+\text { low res. } \mu C T(10)+ \\
\text { von Kossa }(10)+\text { OCT (4) }\end{array}$ & $\begin{array}{l}1 \text { Yucatan mini-swine (3 devices): } \\
\text { high res. } \mu C T(3)+E D X(3)\end{array}$ & \\
\hline 3 years & & $\begin{array}{l}1 \text { Yucatan mini-swine ( } 3 \text { devices): } \\
\text { high res. } \mu C T(3)+E D X(3)\end{array}$ & \\
\hline
\end{tabular}

Figure 1. Study flow chart. Number of animals, total devices and devices assigned to specific analysis methods: time course of degradation and tissue mineralisation (first column), qualitative degradation process (second column) and crystallinity of the degradation product (third column).

\section{QUALITATIVE BACKBONE DEGRADATION ANALYSIS IN DREAMS 1G}

Qualitative backbone degradation in DREAMS $1 \mathrm{G}$ was achieved by element-sensitive X-ray analysis (QUANTAX 200; Bruker AXS GmbH, Karlsruhe, Germany) combined with scanning electron microscopy (EVO LS 15; Carl Zeiss Microimaging $\mathrm{GmbH}$, Göttingen, Germany) at 0, 28, 90 and 180 days and 1, 2 and 3 years for DREAMS $1 \mathrm{G}(\mathrm{n}=5,8,8,8,6,3,3)$. High-resolution micro-computed tomography $(\mu \mathrm{CT})$ scans (resolution: $1.4 \mu \mathrm{m}$ ) (SkyScan 1172; Bruker microCT, Kontich, Belgium) were performed for selected DREAMS $1 \mathrm{G}$ at $0,28,90$ and 180 days and 1,2 and 3 years after implantation ( $n=3$ per time point), following embedding of specimens into PMMA. The Supplementary Appendix contains a detailed description of the qualitative backbone degradation of DREAMS $1 \mathrm{G}$.

\section{TEMPORAL DEGRADATION ANALYSIS OF DREAMS 2G PLANIMETRIC BACKBONE DEGRADATION ANALYSIS}

Explanted DREAMS $2 \mathrm{G}$ samples were analysed by planimetry to assess the extent of backbone degradation at 28, 90 and 180 days ( $n=12$ per time point) and at 1 and 2 years $(n=8$ and $n=10$, respectively). Treated arterial segments were embedded in PMMA, and all struts in scaffold cross-sections close to the proximal and distal ends, as well as one middle section per sample at an interval of approximately $9 \mathrm{~mm}$, were examined under polarised and nonpolarised light with a stereomicroscope (BX51; Olympus GmbH, Hamburg, Germany). A total of 419 struts in 36 sections at 28 days, 395 struts in 36 sections at 90 days, 414 struts in 35 sections at 180 days, 218 struts in 24 sections at 1 year and 250 struts in 30 sections at 2 years were analysed. Single struts were analysed separately under polarised light at a magnification of $100-500 x$. To determine the degree of magnesium conversion into calcium phosphate, the area of non-degraded scaffold material, eliciting a bright signal under polarised light, and the initial strut area were traced using ProgRes ${ }^{\circledR}$ CapturePro, Version 2.8.8 (JENOPTIK AG, Jena, Germany) (Supplementary Figure 1). The percentage of magnesium conversion (\%Ac) was calculated for each scaffold cross-section using the formula $\% A c=\left(1-\sum \mathrm{Au} / \sum \mathrm{Ao}\right) \times 100$ $(\mathrm{Au}=$ unconverted area, $\mathrm{Ao}=$ outer contour of the strut area).

ASSESSMENT OF STRUT DISCONTINUITIES AS A FUNCTION OF TIME

Low-resolution $\mu \mathrm{CT}$ scans (resolution: $6.9 \mu \mathrm{m}$ ) (SkyScan 1172) were performed of all DREAMS $2 \mathrm{G}$ samples at 28 and 90 days as well as at 1 and 2 years after implantation $(n=12,12,8,10)$ following PMMA embedding of treated arteries. Strut discontinuities were defined as complete displacement of strut fragments or 
presence of circumferential fracture lines around struts by $\mu \mathrm{CT}$ analysis. The number and location of strut discontinuities (without including connectors) were manually marked for each scaffold using 3D $\mu \mathrm{CT}$ visualisation and expressed as number of discontinuities per mm scaffold length (discontinuity density).

\section{ASSESSMENT OF CRYSTALLINITY OF DEGRADATION PRODUCT BY X-RAY DIFFRACTION ANALYSIS}

$\mathrm{X}$-ray diffraction (XRD) analysis was performed in three uncoated DREAMS 2G treated vessels of the 180 days cohort and three naive uncoated DREAMS 2G samples (control) using a wide-angle range Bruker D8 diffractometer (Bruker AXS GmbH) to assess the presence of crystalline material phases in a large sample volume.

\section{HISTOPATHOLOGICAL ASSESSMENT OF TISSUE MINERALISATION}

DREAMS $2 \mathrm{G}$ treated vessel sections from 28 days to two years were analysed for tissue mineralisation using von Kossa staining as previously described ${ }^{5}$. Stained mid sections were qualitatively assessed for the presence of mineralised materials in arterial tissue. In addition, myocardial sections in the territory of treated arteries were evaluated for emboli, signs of ischaemia and scarring.

\section{OPTICAL COHERENCE TOMOGRAPHY (OCT)}

Optical coherence tomography was performed for 6/4/4 DREAMS $2 \mathrm{G}$ treated vessels of the 180 days/1-year/2-year cohorts, respectively, following implantation and before sacrifice with the ILUMIEN $^{\text {TM }}$ imaging system (St. Jude Medical GmbH, Eschborn, Germany). Following vasodilatation using nitroglycerine, the OCT catheter was advanced beyond the distal scaffold segment into native distal vessel segments, and the pullback acquired to a point proximal to the scaffold. Scaffold struts were qualitatively investigated for the presence of light reflection (blooming artefact) and translucency as a function of time.

\section{OCT ATTENUATION SCORING}

To examine time-dependent attenuation of OCT light resulting from interaction with tissue components, an ordinal score from 1 to 4 was applied for each stent strut clearly visualised in the OCT pullbacks available at 180 days ( $\mathrm{n}=6$ with 115 frames), 1 year ( $\mathrm{n}=4$ with 66 frames) and 2 years $(\mathrm{n}=4$ with 52 frames); attenuation score 1: strut sharply delineated, complete attenuation behind strut; attenuation score 2: strut diffusely delineated, complete attenuation behind strut; attenuation score 3: strut diffusely delineated, moderate attenuation behind strut; attenuation score 4: strut diffusely delineated, mild attenuation behind strut.

In each stent, every third frame ( \pm 2 frames) containing at least three clearly visible struts was evaluated. Each strut was scored using the score given above. At 180 days, a total of six stents were analysed ( $n=115$ frames, $n=1,359$ struts). At one year $(n=66$ frames, $n=594$ struts) and two years ( $n=52$ frames, $n=278$ struts), four stents were available, respectively. At one year, only three stents matched the visibility criteria and could be evaluated.
In the remaining stents at one year, stent struts were no longer discernible. Analysis was performed at frame level.

\section{STATISTICAL ANALYSIS}

Continuous data are presented as mean with standard deviation. Non-parametric data are presented as median with interquartile range. A Wilcoxon Kruskal-Wallis rank-sum test was used to calculate the significance of differences between medians of nonparametric data. A chi-square value of $\mathrm{p}<0.05$ was considered statistically significant. For frame-level and tissue block-level analysis, a generalised linear mixed regression model (GLMM) was applied to account for the clustered nature of data. Duration of stent implantation was considered as a fixed effect, while animal and frame number were considered as random effects. A linear data structure with normal distribution was assumed. Data are presented as mean with upper and lower 95\% confidence interval. A p-value of $\mathrm{p}<0.05$ was considered statistically significant. To evaluate intra- and inter-observer variability of OCT attenuation score readings and strut discontinuities, the intra-class correlation coefficient (ICC) was calculated. To assess intra-observer correlation, OCT attenuation scores were repeatedly derived after approximately four weeks in two stents at 180 days, two stents at 1 year and two stents at 2 years. Statistical analysis was performed with JMP Pro, Version 12.0 (SAS Institute, Cary, NC, USA) and SPSS, Version 22 (IBM Corp., Armonk, NY, USA).

\section{Results}

\section{QUALITATIVE DEGRADATION ANALYSIS IN DREAMS 1G}

The degradation process was identified as initiating at the strut surface and proceeding towards the inner strut core over time. It occurred in two main phases (Figure 2, panels IA-ID). The Supplementary Appendix and Supplementary Figure 2 contain a detailed description of qualitative degradation in DREAMS $1 \mathrm{G}$.

Co-registration of energy dispersive X-ray analysis (EDX) and high-resolution micro CT was performed to allow a volumetric pseudo colour visualisation of the multi-phase degradation process.

The initial corrosion phase mainly containing oxygen and magnesium was evenly distributed over the entire scaffold, indicating a homogenous macroscopic corrosive attack (Figure 2, panel IIA). At later time points during the intermediate and second phase (Figure 2, panels IIB \& IIC), the degradation process appeared to be heterogeneous until almost full conversion of the backbone. The final calcium and phosphate-rich conversion phase was homogeneously distributed over the entire scaffold footprint (Figure 2, panel IID).

The residual magnesium component decreases along the entire scaffold until complete conversion of the backbone (Figure 2).

\section{DURATION OF MAGNESIUM BIOABSORPTION IN DREAMS 2G}

Planimetric assessment of magnesium degradation revealed $12.1 \%$ (95\% CI: $8.21,15.98)$ degradation at 28 days, $43.8 \%(95 \%$ CI: $39.87,47.65)$ at 90 days, $66.5 \%(95 \%$ CI: 62.73, 70.25) at 180 days, $94.8 \%(95 \%$ CI: $90.22,99.43)$ at 1 year and $99.8 \%$ (95\% CI: 95.68, 103.92) at 2 years $(\mathrm{p}=0.0001)$ (Figure 3 ). 


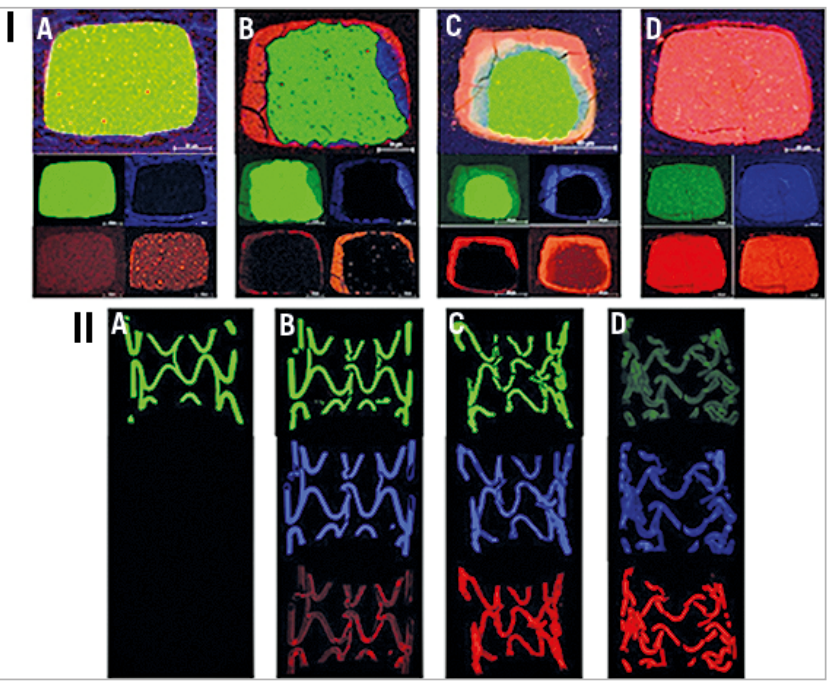

Figure 2. Magnesium backbone degradation process. I) Crosssectional elemental mappings (EDX) of DREAMS $1 G$ using pseudo colour images delineating the two phases of backbone degradation over time: elemental combination images (top) and single elemental mappings of magnesium (green), oxygen (blue), calcium and phosphate (red) (small images below; starting left) following implantation at A) acute, B) 28 days, C) 90 days and D) 180 days.

II) Magnesium backbone volumetric degradation process. Segmented $\mu C T$ pseudo colour images allowing visualisation of volumetric backbone degradation of DREAMS 1G. EDX co-registered and segmented $\mu C T$ attenuation images of one representative scaffold half per time point: magnesium-rich (top, green), oxygen-rich (middle, blue) and calcium/phosphate-rich (bottom, red) scaffold regions. Time points as per

Figure 2-I: A) acute, B) 28, C) 90 and D) 180 days.

\section{SCAFFOLD DISCONTINUITY DENSITY IN DREAMS 2G}

Scaffold discontinuity density was assessed in volumetric lowresolution micro CT images, where all degradation phases, the original magnesium backbone material as well as the intermediate products and amorphous calcium phosphate (ACP) are visible in a single greyscale image. The structural integrity of the scaffold marginally decreased with a low discontinuity density of $0.5 \pm 0.57$ per $\mathrm{mm}$ at 28 days up to $3.20 \pm 1.14$ per $\mathrm{mm}$ at 90 days. Discontinuity density above 7.5 per $\mathrm{mm}$, as seen in samples at one and two years, was only qualitatively assessed (Figure 4).

The inter-observer variability for strut discontinuities assessed by $\mu \mathrm{CT}$ was 0.973 .

\section{CRYSTALLINITY OF THE DEGRADATION PRODUCT IN DREAMS 2G}

The characterisation of crystalline phases was evaluated using X-ray diffraction analysis of uncoated DREAMS $2 \mathrm{G}$ scaffolds at 180 days after implantation. Diffraction analysis revealed absence of peaks, excluding the presence of crystalline phases, and confirmed the predominance of amorphous material phases. These findings are in contrast to the predominance of crystal peaks
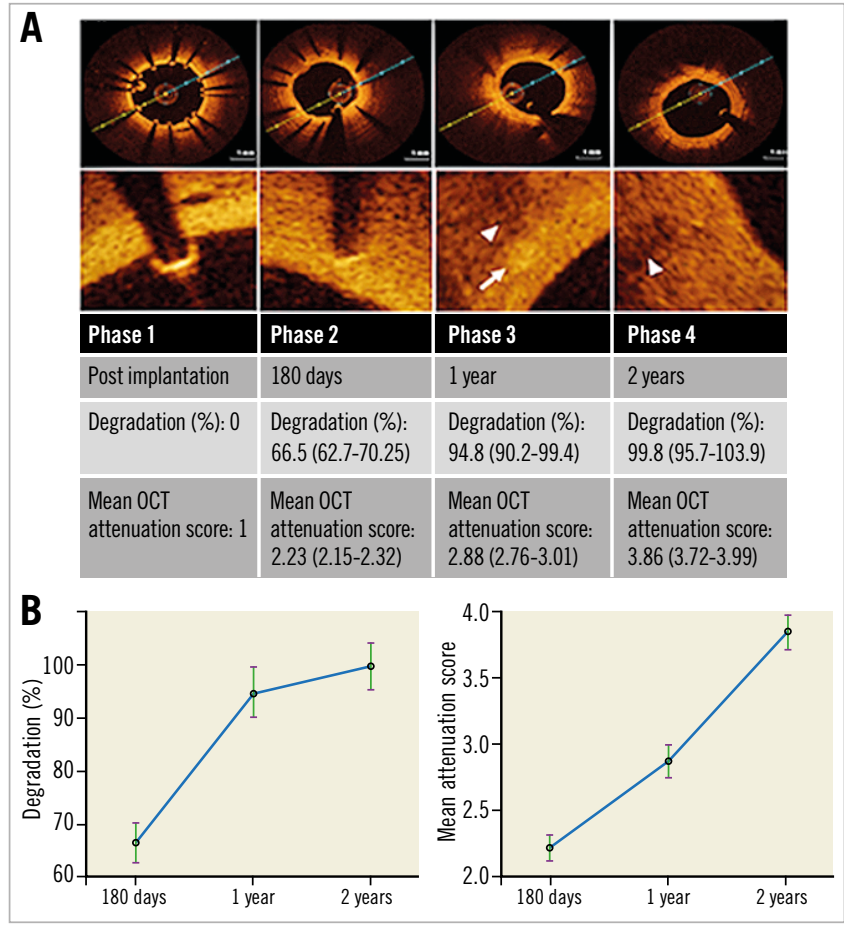

Figure 3. Degradation of DREAMS $2 G$ observed by OCT attenuation score, OCT analysis and planimetric degradation analysis.

A) Changes in strut appearance by OCT are compiled with results from OCT attenuation scoring and planimetric degradation analysis (white arrow indicates residual strut, white arrowheads indicate

strut-associated optical attenuation). B) Comparison of percentage degradation derived by planimetric degradation analysis and OCT attenuation score as a function of time. Statistical analysis is performed on frame-/tissue-block level by generalised linear mixed regression models. Data are presented as mean with upper and lower 95\% confidence interval.

when non-degraded DREAMS 2G control backbone material was examined (Figure 5).

\section{HISTOLOGICAL ASSESSMENT OF TISSUE CALCIUM DEPOSITS IN DREAMS 2G}

Von Kossa stained mid sections of DREAMS 2G-treated arteries were qualitatively analysed for calcium deposits in arterial tissue. Overall, von Kossa staining revealed marked heterogeneity in strut-associated mineralisation, where single struts showed focal spots of mineralisation while others remained unaffected (Figure 6). There was absence of diffusion or embolisation of mineralised material from the immediate strut and peri-strut regions into the deeper surrounding neointimal tissue and myocardial territories, respectively. Signs of myocardial ischaemia and/or scarring were seen in 4/12,3/12,1/12,0/8 and 1/10 DREAMS 2G-treated animals at 28, 90, 180, 365 and 730 days, respectively. Small focal myocardial emboli were observed in one animal of the 180 days cohort; mineralised foreign material was absent in all of the myocardial sections. Since the frequency of myocardial ischaemia/ 


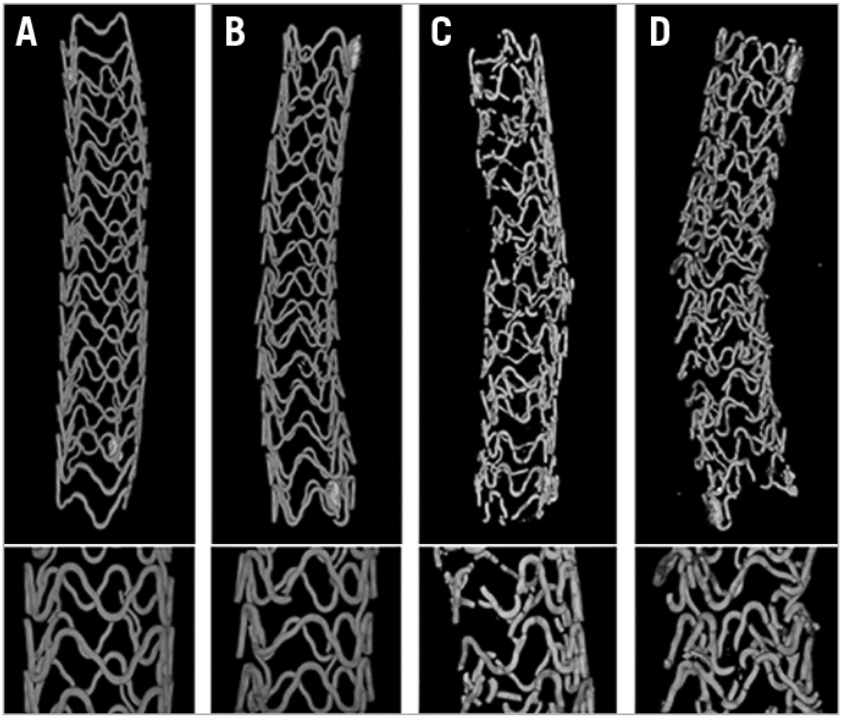

Figure 4. Discontinuity analysis of DREAMS 2G. Representative low-resolution $\mu C T$ images of the entire scaffold at 28 days $(A)$, 90 days $(B)$, one year $(C)$ and two years $(D)$. The original magnesium backbone material as well as the intermediate products and amorphous calcium phosphate are visible. Magnifications of the mid sections are shown in the bottom row.

scarring was higher at time points close to the procedure, periinterventional functional ischaemia is the most likely explanation of this phenomenon.

\section{OCT ATTENUATION SCORE}

A mean score of 2.23 (95\% CI: 2.15, 2.32) at 180 days, $2.88(95 \%$ CI: $2.76,3.01)$ at 1 year and $3.86(95 \%$ CI: $3.72,3.99)$ at 2 years resulted $(\mathrm{p}=0.0001)$.

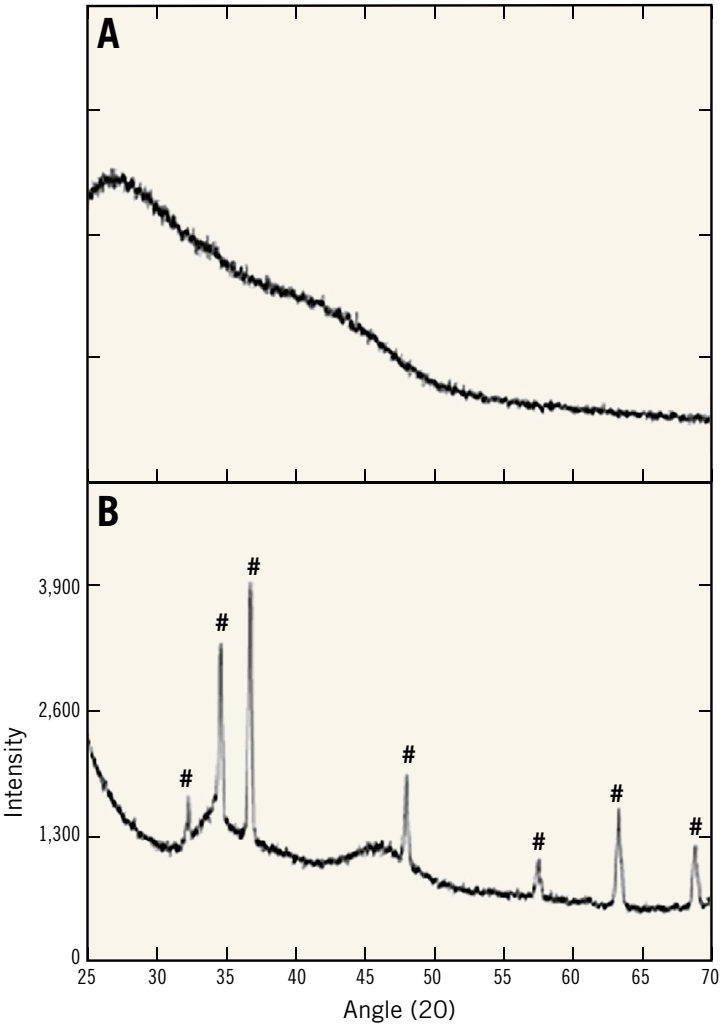

Figure 5. Crystallinity of degradation product. Representative X-ray diffractograms of uncoated DREAMS $2 G$ backbone degradation product at 180 days without crystalline phases (A), and nondegraded DREAMS $2 G$ backbone material with distinct magnesium peaks (\#) (B).

The inter-observer ICC was 0.942 at 180 days, 0.917 at 1 year and 0.853 at 2 years. The intra-observer ICC was 0.788 at 180 days, 0.871 at 1 year and 0.665 at 2 years (Figure 3).

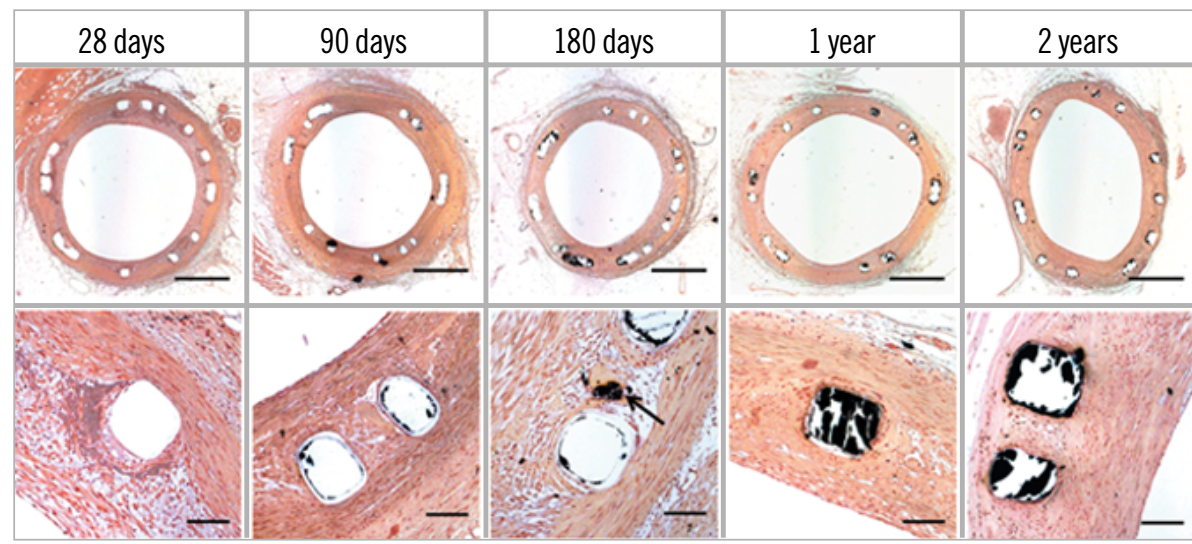

Figure 6. Von Kossa stains. Representative von Kossa stains of DREAMS $2 G$ at $25 x$ (top row) and 200x (bottom row) magnification. Scale bar for 25x magnification is 1,000 $\mu \mathrm{m}$ and for 200x magnification $100 \mu \mathrm{m}$. Marked heterogeneity in strut-associated mineralisation was observed, where single struts showed some mineralisation (arrow) while others remained almost unaffected. As calcium-rich structures are stained in black, the amorphous calcium phosphate can be observed in the area previously occupied by the struts; however, most of the ACP is lost during processing of the slides. Therefore, residual ACP after histopathological processing does not correlate with the overall degree of degradation. 


\section{DESCRIPTIVE COMPARISON OF OCT ATTENUATION SCORE AND PLANIMETRIC STRUT DEGRADATION}

For descriptive comparison of OCT attenuation score and planimetric strut degradation, OCT attenuation score (frame-level data) and planimetric strut degradation (tissue block-level) were plotted as a function of time (Figure 3). The mean percentage of degraded magnesium backbone material increased from 66.5\% (62.7$70.3 \%)$ at six months to $94.8 \%(90.2-99.4 \%)$ at 12 months, while the mean OCT attenuation score also increased from 2.23 (2.15$2.32)$ at six months to $2.88(2.76-3.01)$ at 12 months.

\section{Discussion}

The current study aimed to investigate the qualitative and temporal course of degradation of magnesium alloy-based bioresorbable vascular scaffolds in juvenile swine. In this regard the main findings can be described as follows:

(i) the initial corrosion phase of cross-sectional scaffold struts is characterised by an oxygen- and magnesium-rich layer, which proceeds inwards towards the strut core over time;

(ii) the final degradation product of scaffold struts was identified as amorphous calcium phosphate;

(iii) degradation of DREAMS $2 \mathrm{G}$ magnesium alloy backbone is almost complete at one year after implantation, with a residual non-degraded magnesium area of $5.2 \%$ by cross-sectional planimetric analysis;

(iv) dissemination of strut-associated mineralisation into the surrounding neointimal tissue was not observed and there was absence of myocardial emboli originating from non-embedded scaffold struts, addressing key safety aspects during biodegradation of DREAMS $2 \mathrm{G}$.

\section{TEMPORAL COURSE OF DREAMS 2G DEGRADATION}

Planimetry of resin-embedded stented vascular segments revealed a residual non-degraded magnesium backbone area of $5.2 \%$ at one year, which was accompanied by a rapid increase in discontinuity density between 28 days and one year. Importantly, the absence of mineralised emboli in myocardial sections confirmed the overall safety of DREAMS $2 \mathrm{G}$ degradation over time. The declining solid scaffold core alongside the incremental discontinuity density at one-year follow-up are likely to result in a substantial loss of mechanical integrity and represent the very basis for restoring vascular function in the clinical setting. In the BIOSOLVE II trial, DREAMS $2 \mathrm{G}$ was examined by angiography and intravascular imaging in 123 patients up to 12-month follow-up and showed a good safety profile in the absence of excessive recoil and neointimal growth ${ }^{6}$. Moreover, vasomotion testing performed in a subgroup of patients revealed greater than $3 \%$ diameter change following administration of nitroglycerine and acetylcholine, respectively, confirming the presence of vasomotion at six- and 12-month follow-up ${ }^{6}$. Whether this incremental gain in vasomotion capacity will translate into beneficial clinical outcome remains to be determined in dedicated clinical trials. In this regard, DREAMS $2 \mathrm{G}$ offers the advantage of a rapid pace of degradation, where the almost complete absence of magnesium backbone material was documented after 12 months, which contrasts with the three-year time span of biodegradation in the Absorb ${ }^{\mathrm{TM}}$ BVS (Abbott Vascular, Santa Clara, CA, USA) ${ }^{7}$. Nevertheless, patience to await long-term follow-up and dedicated clinical trials is warranted to answer the question whether this technological difference translates into clinical benefit.

\section{OPTICAL COHERENCE TOMOGRAPHY IMAGING OF DREAMS 2G DEGRADATION}

OCT was identified as a useful imaging tool to delineate strut degradation as a function of time in the current series of animal studies. While freshly implanted DREAMS $2 \mathrm{G}$ struts revealed an optical density reminiscent of metallic DES, sustained degradation caused partial translucence between 180 and 365 days following implantation, which turned into complete translucence at 730 days. In comparison with OCT imaging data of polymeric bioabsorbable vascular scaffolds (Absorb BVS), BVS struts elicit complete translucence in the absence of optical shadowing acutely after implantation, and slowly convert into dissolved black and bright boxes as bioabsorption progresses ${ }^{8}$. Signal attenuation as a result of optical shadowing at the back of stent struts is not observed at any point in time in polymeric BRS, which can be used as a distinguishing feature relative to metallic BRS. Further OCT imaging studies are needed to inform us whether detailed investigation of strut degradation is feasible following implantation of DREAMS $2 \mathrm{G}$.

\section{DEVICE ITERATION AND ASSOCIATION WITH CLINICAL OUTCOME}

DREAMS 2G was developed as an iteration of previous generations of magnesium alloy-based bioresorbable metal scaffolds with the intention to improve upon scaffolding time, early elastic recoil and late efficacy using a novel antiproliferative coating strategy in comparison to its predecessor versions ${ }^{4,9,10}$. In this regard, a substantial increase in degradation time and, consequently, prolonged integrity of the magnesium scaffold could be achieved with the current coating technology ${ }^{11,12}$. In a previous study, Wittchow et al investigated the predecessor version DREAMS $1 \mathrm{G}$ with regard to vascular reactions and degradation in a preclinical animal model and compared its performance to older-generation paclitaxel-eluting stents owing to the release of paclitaxel from a PLGA-polymer matrix in DREAMS $1 \mathrm{G}^{4}$. Angiography, histomorphometry and histopathology data were acquired, showing equivalence to TAXUS ${ }^{\mathrm{TM}}$ Liberté $^{\mathrm{TM}}$ (Boston Scientific, Marlborough, MA, USA) paclitaxel-eluting stents and superiority to eucaTAX (eucatech AG, Weil am Rhein, Germany) paclitaxeleluting stents with regard to late lumen loss, intimal area, fibrin score and endothelialisation. Over the course of time, DREAMS $1 \mathrm{G}$ was investigated in the BIOSOLVE-I first-in-man study, where the magnesium scaffold was proven safe in a total of 46 patients. Target lesion failure was $4 \%$ at six months and $7 \%$ at 12 months ${ }^{11}$, with late loss being greater than what we have been accustomed 
to with newer-generation DES. Because of concerns regarding the toxicity of paclitaxel and too rapid drug release from PLGA polymer, DREAMS $2 \mathrm{G}$ was introduced to overcome the limitations of DREAMS 1G; prolonged degradation kinetics achieved by sustained protection of the magnesium backbone by switching to drug release from a PLLA polymer and the use of sirolimus as antiproliferative drug contributed substantially to a significantly improved clinical outcome in the BIOSOLVE-II trial ${ }^{12}$.

\section{Study limitations}

The current study was performed in non-diseased juvenile porcine coronary arteries, which raises the question as to how many of these preclinical findings can be translated into clinical practice. While important limitations remain with regard to the absence of atherosclerotic disease conditions and the factor of animal growth during experimentation with juvenile pigs, the qualitative aspects of degradation can be reliably assessed. The exact time course of degradation, however, might differ among porcine and human coronary arteries, which warrants confirmation in dedicated clinical trials.

\section{Conclusions}

Degradation of first- and second-generation drug-eluting bioresorbable metal scaffolds entails two different chemical phases resulting in amorphous calcium phosphate as the final degradation product. In porcine coronary arteries, DREAMS $2 \mathrm{G}$ was almost completely degraded after one year. Strut discontinuities are infrequent at 28 days and increase rapidly up to 90 days, with almost complete disintegration of scaffold struts at one year. Optical coherence tomography may be used as a clinical imaging tool to monitor degradation of DREAMS $2 \mathrm{G}$ in man.

\section{Impact on daily practice}

Assessment of degradation kinetics and qualitative characterisation of degradation products remains paramount during preclinical evaluation of bioresorbable vascular scaffolds and is a prerequisite to proceeding to clinical evaluation. Here, we present in-depth analysis of qualitative and temporal biodegradation in first- and second-generation magnesium-based bioresorbable vascular scaffolds, which have recently been adopted in first-inhuman studies with considerable success.

\section{Acknowledgements}

XRD measurements (Daniel Jansen, PhD; Geo-Center of Northern Bavaria, Erlangen, Germany).

\section{Funding}

The study was funded by BIOTRONIK AG, Bülach, Switzerland.

\section{Conflict of interest statement}

P. Ruppelt and P. Zumstein are employees of BIOTRONIK AG.

R. Waksman and M. Joner receive grant research support and consulting fees from Biotronik. M. Haude receives consulting fees from BIOTRONIK. C. Lapointe Corriveau reports personal fees from AccelLAB Inc., other from BIOTRONIK AG, during the conduct of the study; personal fees from AccelLAB Inc., other from BIOTRONIK, outside the submitted work. E. Wittchow reports personal fees from BIOTRONIK, during the conduct of the study. The other authors have no conflicts of interest to declare.

\section{References}

1. Windecker S, Kolh P, Alfonso F, Collet JP, Cremer J, Falk V, Filippatos G, Hamm C, Head SJ, Jüni P, Kappetein AP, Kastrati A, Knuuti J, Landmesser U, Laufer G, Neumann FJ, Richter DJ, Schauerte P, Sousa Uva M, Stefanini GG, Taggart DP, Torracca L, Valgimigli M, Wijns W, Witkowski A. 2014 ESC/EACTS Guidelines on myocardial revascularization. EuroIntervention. 2015; 10:1024-94.

2. Otsuka F, Yahagi K, Ladich E, Kutys R, Alexander R, Fowler D, Virmani R, Joner M. Hypersensitivity reaction in the US Food and Drug Administration-approved second-generation drugeluting stents: histopathological assessment with ex vivo optical coherence tomography. Circulation. 2015;131:322-4.

3. Otsuka F, Byrne RA, Yahagi K, Mori H, Ladich E, Fowler DR, Kutys R, Xhepa E, Kastrati A, Virmani R, Joner M. Neoatherosclerosis: overview of histopathologic findings and implications for intravascular imaging assessment. Eur Heart J. 2015;36:2147-59.

4. Wittchow E, Adden N, Riedmüller J, Savard C, Waksman R, Braune M. Bioresorbable drug-eluting magnesium-alloy scaffold: design and feasibility in a porcine coronary model. EuroIntervention. 2013;8:1441-50.

5. Otsuka F, Kramer MC, Woudstra P, Yahagi K, Ladich E, Finn AV, de Winter RJ, Kolodgie FD, Wight TN, Davis HR, Joner M, Virmani R. Natural progression of atherosclerosis from pathologic intimal thickening to late fibroatheroma in human coronary arteries: A pathology study. Atherosclerosis. 2015;241: $772-82$.

6. Haude M, Ince H, Abizaid A, Toelg R, Lemos PA, von Birgelen C, Christiansen EH, Wijns W, Neumann FJ, Kaiser C, Eeckhout E, Lim ST, Escaned J, Onuma Y, Garcia-Garcia HM, Waksman R. Sustained safety and performance of the second-generation drug-eluting absorbable metal scaffold in patients with de novo coronary lesions: 12-month clinical results and angiographic findings of the BIOSOLVE-II first-in-man trial. Eur Heart J. 2016;37:2701-9.

7. Otsuka F, Pacheco E, Perkins LE, Lane JP, Wang Q, Kamberi M, Frie M, Wang J, Sakakura K, Yahagi K, Ladich E, Rapoza RJ, Kolodgie FD, Virmani R. Long-term safety of an everolimus-eluting bioresorbable vascular scaffold and the cobaltchromium XIENCE V stent in a porcine coronary artery model. Circ Cardiovasc Interv. 2014;7:330-42.

8. Onuma Y, Serruys PW, Perkins LE, Okamura T, Gonzalo N, Garcia-Garcia HM, Regar E, Kamberi M, Powers JC, Rapoza R, van Beusekom H, van der Giessen W, Virmani R. Intracoronary optical coherence tomography and histology at 1 month and 2, 3, and 4 years after implantation of everolimus-eluting bioresorbable 
vascular scaffolds in a porcine coronary artery model: an attempt to decipher the human optical coherence tomography images in the ABSORB trial. Circulation. 2010;122:2288-300.

9. Erbel R, Di Mario C, Bartunek J, Bonnier J, de Bruyne B, Eberli FR, Erne P, Haude M, Heublein B, Horrigan M, Ilsley C, Bose D, Koolen J, Luscher TF, Weissman N, Waksman R; PROGRESS-AMS (Clinical Performance and Angiographic Results of Coronary Stenting with Absorbable Metal Stents) Investigators. Temporary scaffolding of coronary arteries with bioabsorbable magnesium stents: a prospective, non-randomised multicentre trial. Lancet. 2007;369:1869-75.

10. Waksman R, Erbel R, Di Mario C, Bartunek J, de Bruyne B, Eberli FR, Erne P, Haude M, Horrigan M, Ilsley C, Böse D, Bonnier H, Koolen J, Lüscher TF, Weissman NJ; PROGRESSAMS (Clinical Performance Angiographic Results of Coronary Stenting with Absorbable Metal Stents) Investigators. Early- and long-term intravascular ultrasound and angiographic findings after bioabsorbable magnesium stent implantation in human coronary arteries. JACC Cardiovasc Interv. 2009;2:312-20.

11. Haude M, Erbel R, Erne P, Verheye S, Degen H, Böse D, Vermeersch P, Wijnbergen I, Weissman N, Prati F, Waksman R, Koolen J. Safety and performance of the drug-eluting absorbable metal scaffold (DREAMS) in patients with de-novo coronary lesions: 12 month results of the prospective, multicentre, first-inman BIOSOLVE-I trial. Lancet. 2013;381:836-44.
12. Haude $M$, Ince $H$, Abizaid A, Toelg R, Lemos PA, von Birgelen C, Christiansen EH, Wijns W, Neumann FJ, Kaiser C, Eeckhout E, Lim ST, Escaned J, Garcia-Garcia HM, Waksman R. Safety and performance of the second-generation drug-eluting absorbable metal scaffold in patients with de-novo coronary artery lesions (BIOSOLVE-II): 6 month results of a prospective, multicentre, non-randomised, first-in-man trial. Lancet. 2016;387:31-9.

\section{Supplementary data}

Supplementary Appendix. Qualitative backbone degradation analysis in DREAMS $1 \mathrm{G}$.

Supplementary Figure 1. Planimetric degradation analysis. Microscopic images of the scaffold cross-section with unpolarised light (A, 25x magnification), of a single strut showing an unconverted core $(\mathrm{B}, 500 \mathrm{x}$ magnification) and the outer enclosure of the strut area (Ao) and the unconverted area ( $\mathrm{Au}$ ) using the software program ProgRes ${ }^{\circledR}$ CapturePro 2.8.8 (C).

Supplementary Figure 2. Main degradation phases. Simplified chemical process equations representing corrosion phase (a), conversion phase (b) and magnesium absorption (c) of the overall degradation process.

The supplementary data are published online at:

http://www.pcronline.com/

eurointervention/142nd_issue/187 


\section{Supplementary dataSupplementary Appendix. Qualitative backbone degradation analysis in DREAMS 1G}

\section{Scanning electron microscopy (SEM) and energy dispersive $X$-ray analysis (EDX)}

Qualitative backbone degradation was examined by element-sensitive X-ray analysis

(QUANTAX 200; Bruker AXS GmbH, Karlsruhe, Germany) combined with scanning electron microscopy (EVO LS 15; Carl Zeiss Microimaging GmbH, Göttingen, Germany) at 0, 28, 90 and 180 days and 1, 2 and 3 years for DREAMS $1 G(n=5,8,8$, $8,6,3,3)$. SEM was used as a two-dimensional atomic weight-sensitive imaging technique (backscatter detector) on cross-sections of coronary arteries embedded in polymethyl methacrylate (PMMA). EDX was further used for elemental characterisation of degradation products and for two-dimensional mapping and subsequent qualitative comparison of the examined time points.

\section{Volumetric degradation analysis by micro-computed tomography $(\mu C T)$}

High-resolution $\mu \mathrm{CT}$ scans (resolution: $1.4 \mu \mathrm{m}$ ) (SkyScan 1172; Bruker microCT, Kontich, Belgium) were performed for selected DREAMS 1G at 0, 28, 90 and 180 days and 1, 2 and 3 years after implantation ( $n=3$ per time point), following embedding of specimens into PMMA. Scaffold data sets were segmented into 3D pseudo colour images using attenuation coefficients and co-registered with existing EDX data to analyse the volumetric distribution of degradation products. For this purpose, elemental EDX mappings of calcium and phosphate (combined), magnesium and oxygen were co-registered with specific attenuation ranges in $\mu \mathrm{CT}$ data sets and graphically superimposed with the corresponding $\mu \mathrm{CT}$ cross-sections.

\section{Qualitative degradation analysis in DREAMS $1 G$}

The degradation process was identified as initiating at the strut surface and proceeding towards the inner strut core over time. It occurred in two main phases (Figure 2, panels IA-1D).

The initial corrosion phase (first phase) was characterised by the presence of an oxygen- and magnesium-rich layer, chemically specified by a reaction of magnesium 
and water to magnesium hydroxide. In an intermediate phase, a phosphate-enriched layer was identified adjacent to the initial corrosion layer (Figure 2, panels IB \& IC), chemically specified by a reaction of magnesium hydroxide and hydrogen phosphate to magnesium (hydrogen) phosphate as an intermediate step, reacting to amorphous calcium phosphate (ACP) in the presence of calcium ions in the second phase (Supplementary Figure 2). ACP is generated either by substitution of magnesium by calcium in magnesium (hydrogen) phosphate or via direct reaction from magnesium hydroxide with hydrated phosphate and calcium ions (Figure 2, panel ID).

Co-registration of EDX and high-resolution micro CT was performed to allow a volumetric pseudo colour visualisation of the multi-phase degradation process.

The initial corrosion phase mainly containing oxygen and magnesium was evenly distributed over the entire scaffold, indicating a homogenous macroscopic corrosive attack (Figure 2, panel IIA). At later time points during the intermediate and second phase (Figure 2, panels IIB \& IIC), the degradation process appeared to be heterogeneous until almost full conversion of the backbone. The final calcium and phosphate-rich conversion phase was homogeneously distributed over the entire scaffold footprint (Figure 2, panel IID).

The residual magnesium component decreases along the entire scaffold until complete conversion of the backbone (Figure 2). 


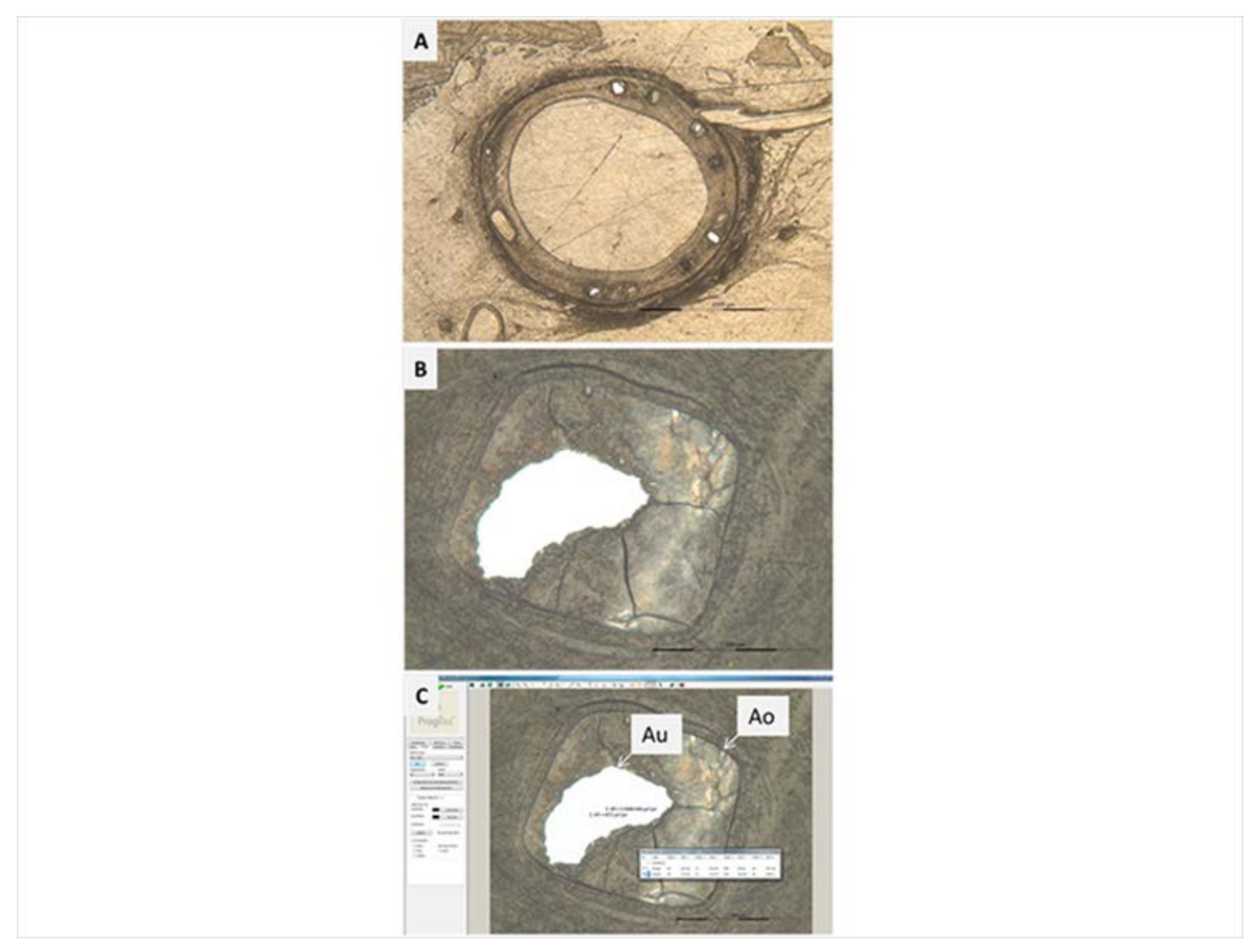

Supplementary Figure 1. Planimetric degradation analysis.

Microscopic images of the scaffold cross-section with unpolarised light (A, 25x

magnification) of a single strut showing an unconverted core (B, 500x magnification) and the outer enclosure of the strut area (Ao) and the unconverted area ( $\mathrm{Au})$ using the software program ProgRes ${ }^{\circledR}$ CapturePro 2.8.8 (C). 


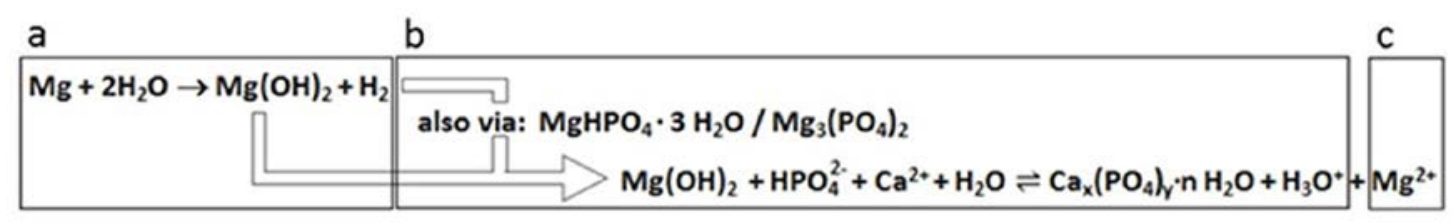

Supplementary Figure 2. Main degradation phases.

Simplified chemical process equations representing corrosion phase (a), conversion phase (b) and magnesium absorption (c) of the overall degradation process. 